\title{
ELECTROCHEMICALLY CONTROLLED RELEASE OF DEXAMETHASONE FROM CONDUCTING POLYMER POLYPYRROLE COATED ELECTRODE
}

by

\section{Reecha Wadhwa}

B.Tech, Indian Institute of Technology, New Delhi, India, 2003

M.Tech, Indian Institute of Technology, New Delhi, India, 2003

\author{
Submitted to the Graduate Faculty of \\ School of Engineering in partial fulfillment \\ of the requirements for the degree of \\ Master of Science
}

University of Pittsburgh 


\title{
UNIVERSITY OF PITTSBURGH \\ SCHOOL OF ENGINEERING
}

This thesis was presented

by

\author{
Reecha Wadhwa
}

It was defended on

October 27, 2006

and approved by

Dr Xinyan Tracy Cui, Assistant Professor, Department of Bioengineering

Dr Carl Lagenaur, Associate Professor, Department of Neurobiology

Dr Kacey Marra, Assistant Professor, Department of Bioengineering

Dr William Wagner, Associate Professor, Department of Bioengineering

Thesis Advisor: Dr Xinyan Tracy Cui, Assistant Professor, Department of Bioengineering 


\title{
ELECTROCHEMICALLY CONTROLLED RELEASE OF DEXAMETHASONE FROM CONDUCTING POLYMER POLYPYRROLE COATED ELECTRODE
}

\author{
Reecha Wadhwa, M.S. \\ University of Pittsburgh, 2006
}

Chronic recordings from micromachined neural electrode arrays often fail a few weeks after implantation primarily due to the formation of an astro-glial sheath around the implant. We propose a drug delivery system, from conducting polymer $(\mathrm{CP})$ coatings on the electrode sites, to modulate the inflammatory implant-host tissue reaction. In this study, polypyrrole (PPy) based coatings for electrically controlled and local delivery of the ionic form of an anti-inflammatory drug, dexamethasone (Dex), was investigated. The drug was incorporated in PPy via electropolymerization of pyrrole and released in PBS using cyclic voltammetry (CV). FTIR analysis of the surface showed the presence of Dex and polypyrrole on the coated electrode. The thickness of the coated film was estimated to be $\sim 50 \mathrm{~nm}$ by ellipsometry. We were able to release $0.5 \mu \mathrm{g} / \mathrm{cm}^{2}$ Dex in $1 \mathrm{CV}$ cycle and upto $92 \%$ Dex after $30 \mathrm{CV}$ cycles. In-vitro studies and immunocytochemistry on murine glial cells suggest that the released drug lowers the count of reactive astrocytes to the same extent as the added drug. In addition, the released drug is not toxic to neurons as seen by healthy neuronal viability in the released drug treated cells. 
TABLE OF CONTENTS

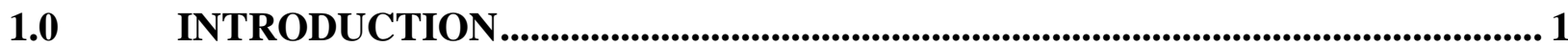

2.0 INCORPORATION OF DEX IN PPY-DEX FILMS ON GOLD ELECTRODES4

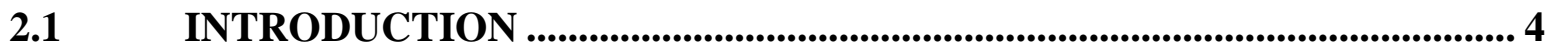

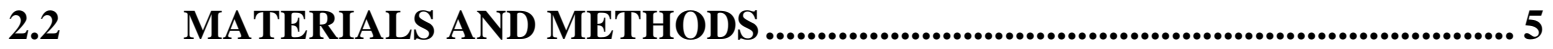

2.2.1 Electrodeposition of Polypyrrole-Dex Coating ................................................ 5

2.2.1.1 Electrode preparation ............................................................................... 5

2.2.1.2 Electrochemical deposition ......................................................................... 5

2.2.2 Fourier Transform Infra Red Spectroscopy (FTIR) ..................................... 6

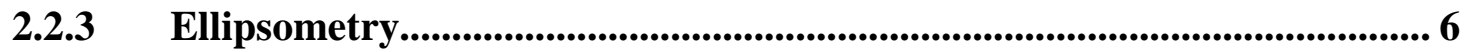

2.2.4 Atomic Force Microscopy (AFM).......................................................... 7

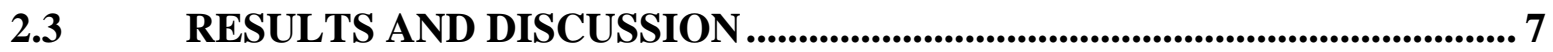

2.4 CONCLUSION ........................................................................................................ 9

D.0 DEXAMETHASONE RELEASE STUDIES.................................................. 13

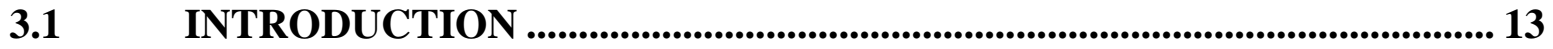

3.2 MATERIALS AND METHODS ............................................................... 14

3.2.1 Scanning Electron Microscopy (SEM).......................................................... 14

3.2.2 Optical Microscopy .............................................................................................. 14

3.2.3 Cyclic Voltammetry ….......................................................................................... 14 
3.2.4 Ultraviolet (UV) Spectroscopy ..................................................................... 15

3.2.5 Impedance Measurements........................................................................... 15

3.2.6 Statistical Analysis .......................................................................................... 16

RESULTS AND DISCUSSION ............................................................... 16

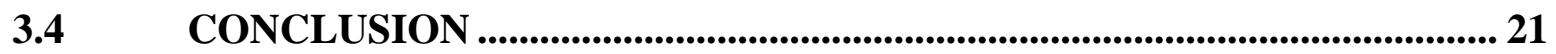

4.0 IN VITRO CELL CULTURE STUDIES .................................................................... 27

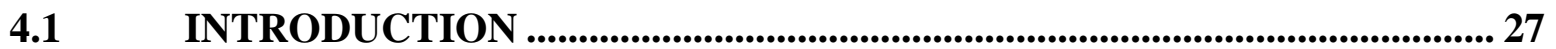

4.2 MATERIALS AND METHODS ................................................................ 27

4.2.1 Primary neurons and astrocytes cell culture................................................. 27

4.2.2 Immunocytochemistry ....................................................................................... 28

4.2.3 Imaging and analysis ................................................................................... 29

4.2.4 Statistical analysis ............................................................................................ 30

4.3 RESULTS AND DISCUSSION ........................................................................ 30

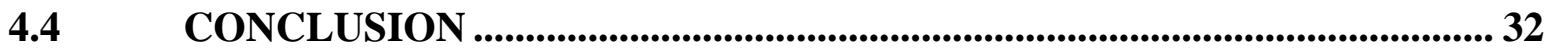

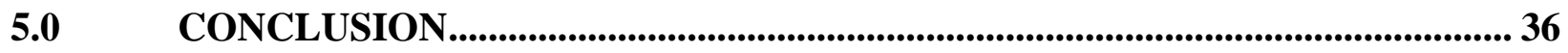

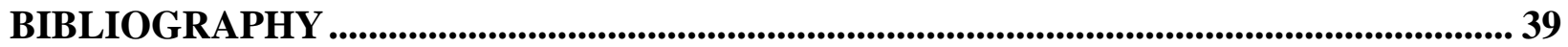




\section{LIST OF FIGURES}

Figure 1. Principle of electropolymerization of polypyrrole. ......................................... 10

Figure 2. Chemical Structure of disodium salt of dexamethasone ........................................ 10

Figure 3. FTIR spectrum of the PPy-Dex polymer coatings on electrode.............................. 11

Figure 4. AFM images for the PPy-Dex coated electrode surface. .................................... 12

Figure 5. Characteristic Cyclic Voltammogram........................................................... 22

Figure 6. Release profile of Dex from the polymer-drug coated electrodes............................. 23

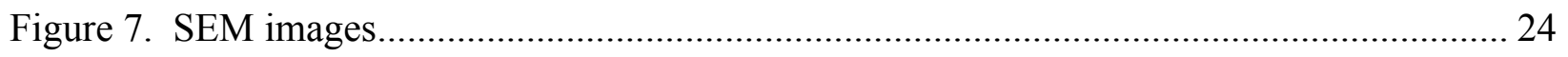

Figure 8. Enface image of the gold polymer interface under optical microscope .................... 25

Figure 9. Impedance spectroscopy for gold and PPy-Dex film coated electrode.................... 26

Figure 10. In-vitro studies for the effect of Dex on reactive astrocytes number ..................... 33

Figure 11. In-vitro studies for the effect of released Dex on astrocytes and microglia............. 34

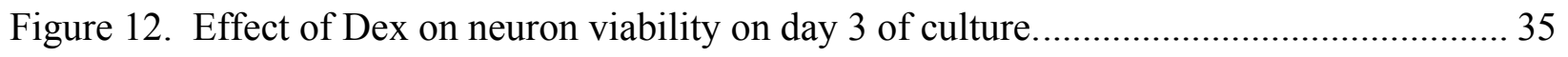




\subsection{INTRODUCTION}

Neural prostheses are implantable microelectronic devices used to bypass, repair or restore damaged neurological function by electrical stimulation or recording from the peripheral and central nervous systems. The chronic application of these devices has been limited by their unpredictable failure over extended periods. Although the precise mechanism for this failure is not completely known, it is suggested that an undesirable brain tissue reaction to the device leads to the formation of a glial scar which then serves as a barrier to neural signal transduction [1-8].

Biomaterials strategies are being explored to minimize or eliminate the undesired tissue reaction. Two major directions are being taken, one being enhancing neuronal growth and adherence to the electrodes via immobilization of biomolecules on electrode surface or through release of growth factors or chemo-attractants from the electrodes[9-17], and the other being mitigating the brain microenvironment through delivery of anti-inflammatory factors in the vicinity of the implant. Dexamethasone (Dex) is a synthetic glucocorticoid anti-inflammatory drug which can reduce inflammation in the Central Nervous System (CNS). The drug is believed to act through its glucocorticoid receptors found in most neurons and glial cells throughout the brain [18]. These receptors' pathways are involved in the inhibition of astrocyte proliferation [19] and microglial activation [20]. Additionally, as an anti-inflammatory substance, Dex reduces numerous pro-inflammatory cytokines, including IL-1 $\beta$, IL-6, INF- $\gamma$ and TNF- $\alpha$ [21]. Peripheral injections of Dex have been shown to effectively minimize the reactive 
tissue response to neural implants in the rat [22]. However, systemic injections have the obvious disadvantage of exposing the whole body to high dosages of the drug and its potential toxicity. Local delivery of drugs at the implant/tissue interface would be most effective. Dexamethasone eluting coatings are being developed [23, 24]. The release of Dex from these coatings, indeed, decreased the degree of reactive tissue response of the brain implants as shown in immunohistopathological studies. However these passive drug eluting coatings can not last as long as needed in applications such as cortical prostheses nor can they release appropriate doses in response to the dynamic change of the local biochemical environment. A microfluidics-based drug injection system is also being investigated for the same purpose, wherein intracortical probes with microfluidic channels are fabricated and tested to understand the possibility of using diffusion-mediated delivery to deliver therapeutic agents into the immediate surroundings of the implanted devices [25]. Convective delivery is also plausible and this method can theoretically incorporate components such as pumps and valves for active control of the release. However, the current fabrication technology has not demonstrated the precision needed for sub-picoliter delivery. Furthermore, adding more components to the neural electrode arrays means more processing steps, more failure points and less reliability.

Conducting polymers have the ability to undergo reversible red-ox reaction under the stimulus of an electrical potential. The doped polymer film can be electrically switched between the oxidized state and reduced state [26]. The red-ox reaction involves charging and discharging of the polymer and is also accompanied by the movement of hydrated ions in and out of the bulk. Utilizing this feature, efforts have been made to develop delivery systems in which conducting polymers can be loaded with some chemical substances (drug, growth factors) and the loaded dopants can be released in response to electrical stimulus. The anionic drugs salicylaten, 2- 
ethylhexylphosphate (EHP) and naproxen and the cationic drug dopamine have been incorporated into conducting polymer membrane and released by electrical potential stimulus in a controlled way [27-29]. Adenosine triphosphate (ATP) was shown to be released from polypyrrole upon sensing bioactive molecules in the solution [30].

Polypyrrole and its derivatives are the most widely used conducting polymers in biomedical applications due to their biocompatibility and customizability [31-36]. Polypyrrole has been electrically deposited onto the electrode site of the neural recording arrays together with CDPGYIGSR, a peptide fragment from laminin $[9,37]$, to improve the neuron-electrode connection in vivo.

In the current study, polypyrrole (PPy) based electrode coating for controlled and local delivery of the ionic form of an anti-inflammatory drug, dexamethasone (Dex), was investigated. The drug incorporation was done using a one-step electropolymerization. The release of the drug was carried out using cyclic voltammetric stimulus and was found to be precisely controllable i.e. the released amount was linear to the number of applied stimuli. The released dexamethasone showed anti-inflammatory effects by lowering number of reactive astrocytes in vitro. In primary neuronal culture, healthy neuronal growth was observed in the drug treated cultures, indicating that the delivery mechanism does not produce any toxic leachables and that the drug does not damage neurons at this dosage. 


\subsection{INCORPORATION OF DEX IN PPY-DEX FILMS ON GOLD ELECTRODES}

\section{$2.1 \quad$ INTRODUCTION}

The first sets of experiments were conducted to incorporate the drug in the polymer films on the model gold electrodes. Gold coated coverslips were prepared and PPy-Dex films were deposited using one step electropolymerization. Upon deposition the films were analyzed using Fourier Transform Infrared Spectroscopy (FTIR), Ellipsometry and Atomic Force Microscopy (AFM). Details of these experiments are described in this chapter. 


\subsection{MATERIALS AND METHODS}

\subsubsection{Electrodeposition of Polypyrrole-Dex Coating}

\subsubsection{Electrode preparation}

$22 \mathrm{~mm}$ plastic coverslips (Fisher) were cut to uniform dimension of $7 \mathrm{~mm}$ x $22 \mathrm{~mm}$, cleaned with $8 \mathrm{~N} \mathrm{HNO}_{3}$ for $30 \mathrm{~min}$, washed in deionized $\mathrm{H}_{2} \mathrm{O}$ and stored in ethanol. In order to create a conductive surface for electrochemical deposition, the cut and cleaned coverslips were sputter coated with a layer of gold, using a Cressington Sputter Coater. The thickness of the gold layer was approximately $40 \mathrm{~nm}$. The gold coated coverslips (electrodes) were stored in a desiccator.

\subsubsection{Electrochemical deposition}

PPy / Dex film was grown potentiostatically on the gold coated coverslips. A two electrode set up was used. The electrochemical cell was a $2 \mathrm{ml}$ glass cuvette containing a working electrode (gold coated coverslip with or without the polymer-drug coatings) and a platinum counter electrode. The coating process was controlled using the Gamry Potentiostat, FAS2/Femostat (Gamry Instruments) with Gamry Framework software. The deposition solution (1 ml) contained 0.1 M pyrrole (Sigma) and 0.1 M dexamethasone disodium phosphate (Sigma). The actual electrode area immersed in the solution was $\sim 0.77 \mathrm{~cm}^{2}$, which is the area covered by the resulting polymer-drug film. In the potentiostatic mode, a constant potential of $1.8 \mathrm{~V}$ relative to the counter was used. The amount of material deposited on the electrode surface was controlled 
by time via the total charge passed during deposition. A range of deposition charge densities (15 $-120 \mathrm{mC} / \mathrm{cm}^{2}$ ) were investigated. $25-30 \mathrm{mC} / \mathrm{cm}^{2}$ was found to be optimal in terms of film stability and release efficiency, and was therefore selected for the rest of the study.

\subsubsection{Fourier Transform Infra Red Spectroscopy (FTIR)}

IR spectroscopic measurements were carried out using the Continnum IR microscope (ThermoNicolet). The detector was cooled with liquid nitrogen before use and the entire system was purged with in-house air at a flow rate of about 25 standard cubic feet per hour. The coated coverslips were placed on the microscope stage and the reflective IR spectrum was collected. The samples were scanned 200 times in the range of $1000-4000 \mathrm{~cm}^{-1}$. The spectrum of a clean gold coverslip was used as the background. The spectra were analyzed using the software OMNIC.

\subsubsection{Ellipsometry}

The PPy-Dex film thickness was measured with a Gaertner L-117 Null ellipsometer. A linearly polarized He-Ne laser with wavelength $632.8 \mathrm{~nm}$ was used in the measurement at incident angle $70^{\circ}$. The analyzer (A) can detect the change in the phase angle $(\Delta)$ and the amplitude change $(\Psi)$ of the light after reflection from the sample. Refractive indices of $n_{1}=1$ for air, $n_{2}=1.45$ [38] for the organic molecular monolayer, and $n_{3}=0.166+3.22 \mathrm{i}$ for gold were used to calculate the thickness of the films using the equation, $\rho=\frac{r_{p}}{r_{s}}=\tan \psi \exp (i \Delta)$ 
where $\rho$ is the ratio of Fresnel Reflection Coefficients $r_{p}$ and $r_{s}$ and within it contains the thickness term.

\subsubsection{Atomic Force Microscopy (AFM)}

Information about the sample surface topography was obtained with Atomic Force Microscopy (AFM) and Scanning Electron Microscopy (SEM). AFM was performed with a MFP3D ${ }^{\mathrm{TM}}$ inverted optical model Atomic Force Microscope (AFM) (Asylum Research, Santa Barbara, CA). For each condition, four samples were used and eight images per sample taken. The images obtained consisted of 256X256 arrays of height data over a scan size of $10 \mu \mathrm{m}$ and a scan rate of $1 \mathrm{~Hz}$. Data were collected in tapping mode using a cantilever tip with height of $15-20 \mu \mathrm{m}$ and radius of $<10 \mathrm{~nm}$. Surface scans from the AFM were analyzed using IGOR Pro5.03 (Wavemetrics, OR, U.S.A) and MFP-3D Xop software version 050126.

\subsection{RESULTS AND DISCUSSION}

A one step electropolymerization was used to deposit films of PPy and Dex on the surface of gold coated electrodes. The underlying principle of electropolymerization of PPy is shown in figure 1 . In presence of a dopant $\mathrm{A}^{-}$(anions or negatively charged biomolecules or drugs), the monomers become electrically oxidized and polymerized and the resulting polymer gets deposited on the anode. Because the polymer backbone is positively charged, the anionic dopant molecule is incorporated to maintain charge neutrality. In our case the dopant was dexamethasone disodium phosphate (Dex), a synthetic glucocorticoid which acts as a pro-drug 
and can get converted into the active dexamethasone [41]. The chemical structure of the prodrug is shown in figure 2. The presence of a phosphate group on the dexamethasone steroid ring structure imparts a negative charge to the drug, which can then be incorporated as a dopant in PPy following electropolymerization.

The deposited film was characterized qualitatively by FTIR spectroscopy. The result is presented in figure 3. From 1800 to $4000 \mathrm{~cm}^{-1}$, the spectrum showed a continuous increase in absorption. This is the tail of the $\sim 1 \mathrm{eV}\left(\sim 8066 \mathrm{~cm}^{-1}\right)$ bipolaron absorption band, which is the signature for electrical conductivity of conducting polymers [42, 43]. The broad band around $1550 \mathrm{~cm}^{-1}$ is the characteristic absorption frequency for double bond vibration of the pyrrole ring $[9,37,44,45]$. Proof of the presence of dexamethasone in the deposited film is clearly seen by the appearance of peaks at $3200-3500 \mathrm{~cm}^{-1}$ (hydroxyl), 2850-3000 $\mathrm{cm}^{-1}$ (methyl) and 1665-1760 $\mathrm{cm}^{-1}$ (carbonyl). These groups can be identified in the chemical structure of dexamethasone.

The thickness of the deposited film was detected using ellipsometry. The average thickness was found to be $50 \mathrm{~nm}$ for the films deposited at charge density of $\left(\sim 30 \mathrm{mC} / \mathrm{cm}^{2}\right)$. The surface morphology of the PPy-Dex was revealed by tapping mode AFM as shown in figure 4. For a deposition charge density of $30 \mathrm{mC} / \mathrm{cm}^{2}$, the films appear smooth. The root mean square roughness (RMS) value was $12 \mathrm{~nm}$. Increasing the deposition charge density to about $120 \mathrm{mC} / \mathrm{cm}^{2}$ resulted in a drastic increase in surface roughness. The RMS value was measured to be about $95 \mathrm{~nm}$. The films deposited at higher charge density did not appear to be uniform, with some areas being rougher or smoother than others. 


\subsection{CONCLUSION}

PPy-Dex films were successfully grown on gold coated coverslips using electrochemical deposition techniques. The incorporation of the drug in the polymer films was confirmed by surface detection, of Dex in PPy-Dex films, using FTIR. The films were further characterized using ellipsometry and AFM analysis. 


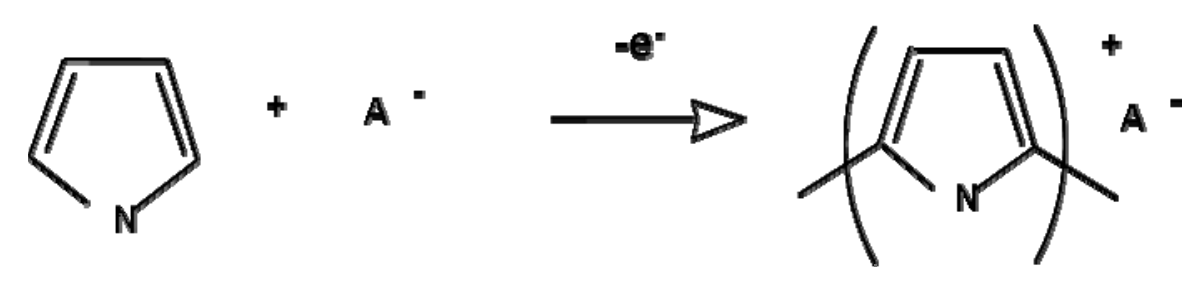

Figure 1. Principle of electropolymerization of polypyrrole.

The negatively charged dopant (A-) is incorporated into the positively charged polymer backbone to maintain electrical neutrality.

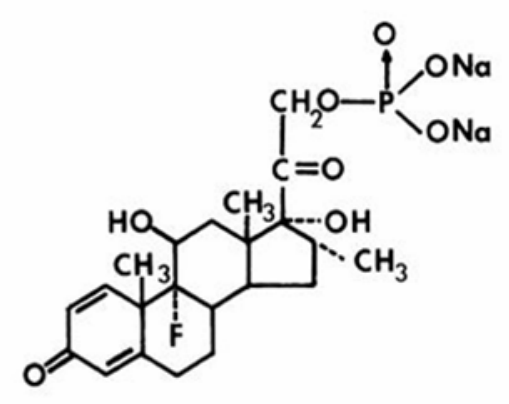

Figure 2. Chemical Structure of disodium salt of dexamethasone 


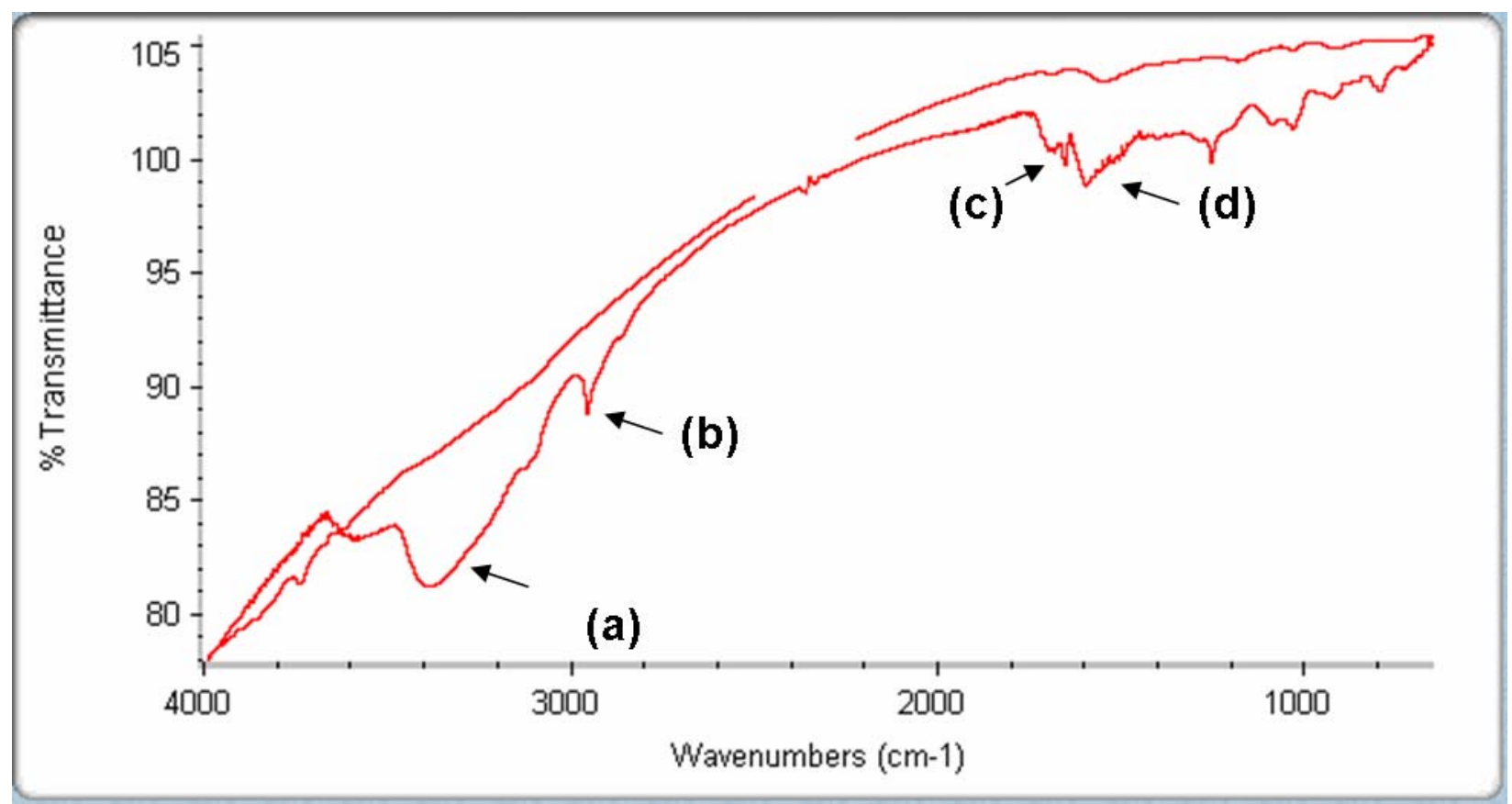

Figure 3. FTIR spectrum of the PPy-Dex polymer coatings on electrode.

The presence of Dex on the surface is indicated by peaks at (a) $3200-3500 \mathrm{~cm}^{-1}$ (hydroxyl), (b) $2850-3000 \mathrm{~cm}^{-1}$ (methyl) and (c) $1665-1760 \mathrm{~cm}^{-1}$ (carbonyl) in the spectrum. No such peaks were obtained in the $\mathrm{PPy}_{-} \mathrm{Cl}^{-}$films, as seen in the second curve. Characteristic broad peak for polypyrrole appears at (d) $1550-1650 \mathrm{~cm}^{-1}$. 

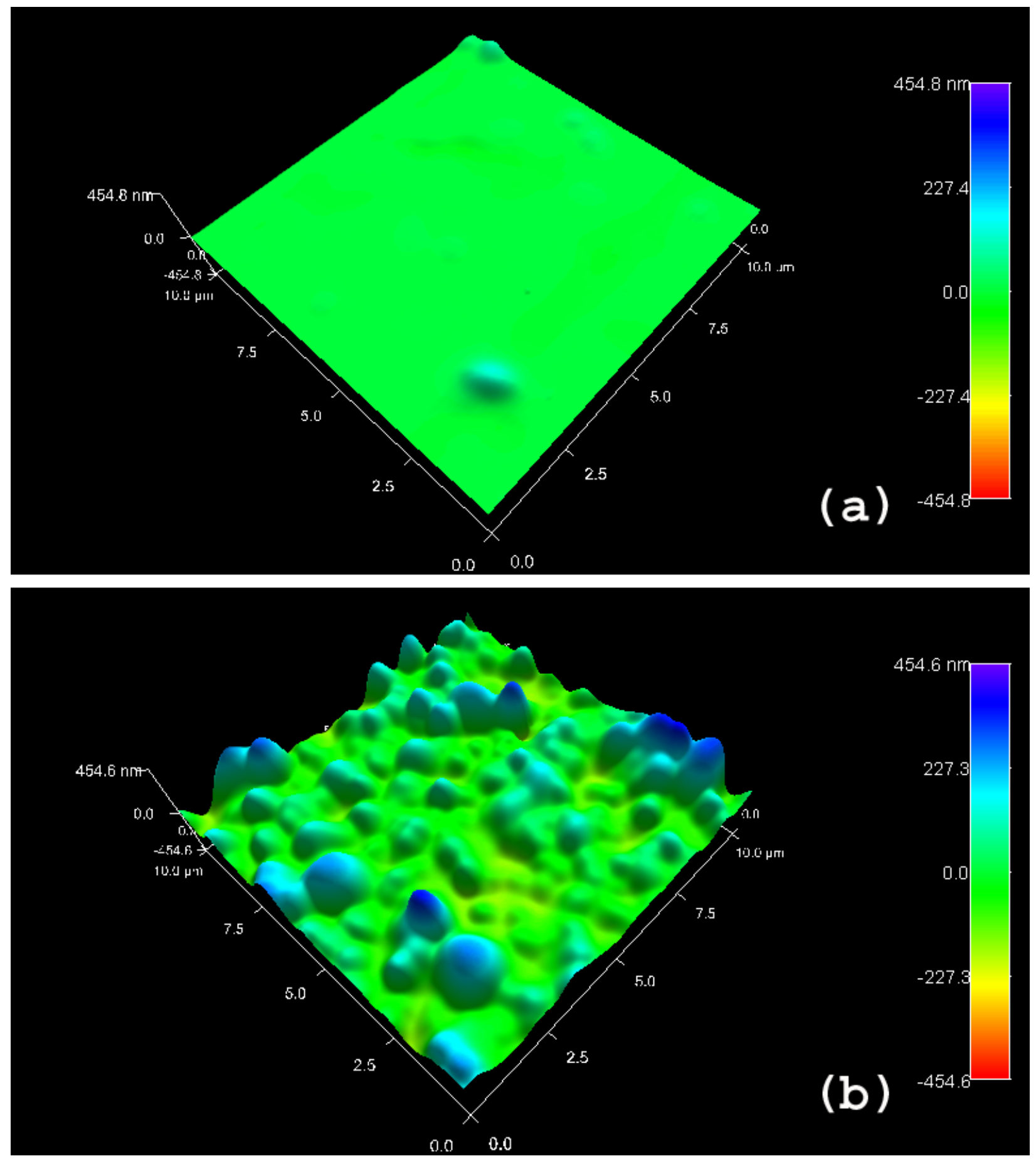

Figure 4. AFM images for the PPy-Dex coated electrode surface.

(a) $30 \mathrm{mC} / \mathrm{cm}^{2}$ (RMS $\sim 12 \mathrm{~nm}$ ) and (b) $120 \mathrm{mC} / \mathrm{cm}^{2}$ (RMS $95 \mathrm{~nm}$ ). As the electropolymerization time increases, the film becomes rougher. 


\subsection{DEXAMETHASONE RELEASE STUDIES}

\subsection{INTRODUCTION}

The previous experiments established that dexamethasone was indeed deposited in the PPy-Dex films on the electrodes. The next logical step was to study the amount of dex released upon application of electrical stimuli. Cyclic voltammetry (CV) was the chosen type of stimuli, wherein the potential was swept between positive and negative values periodically. The film surface before and after release was analyzed by scanning electron microscopy (SEM) and optical microscopy. Impedance measurements were used to evaluate the electrical behavior of the film. The released drug was quantified using ultraviolet spectroscopy. The details of these experiments are described in this chapter. 


\subsection{MATERIALS AND METHODS}

\subsubsection{Scanning Electron Microscopy (SEM)}

The PPy/Dex film surface morphology before and after drug release was studied under SEM. A JEOL 9335 Field Emission Gun SEM was used with a typical voltage of $10 \mathrm{kV}$. A thin film of gold/ palladium $(3.5 \mathrm{~nm})$ was sputtered onto the specimens using a Cressington Sputter Coater, before the surfaces were viewed.

\subsubsection{Optical Microscopy}

An optical microscope (Axioskope 2 MAT, Carl Zeiss) was employed to visualize the changes in morphology of the film after drug release. The images were taken using AxioCamMRC5 Camera (Carl Zeiss) attached to the scope. The images were taken and analyzed using AxioVision Rel 4.2 software.

\subsubsection{Cyclic Voltammetry}

Cyclic voltammetry (CV) was performed using Gamry Potentiostat, FAS2/Femostat (Gamry Instruments) under the software control of Gamry Framework For drug release studies, the CV was done in the two electrode cell described in 2.1.2 and the electrolyte was PBS $(0.1 \mathrm{M}, \mathrm{pH}$ 7.2). The voltage was swept from $-0.8 \mathrm{~V}$ to $+1.4 \mathrm{~V}$ at a scan rate of $100 \mathrm{mV} / \mathrm{s}$. Dex release was quantified by UV absorbance (detail see 2.5) after every five cycles till thirty cycles. Before 
beginning $\mathrm{CV}$, the coated electrodes were soaked in distilled water to remove any loosely attached dexamethasone on the surface. This ensured that the release of Dex is caused mainly by the cyclic potential stimulus. A control was set up, in which the coated electrode was simply soaked in the PBS solution without electrical connection. UV reading of the solution was taken six successive times at time intervals of five minutes, to compare with the six sets of five CV cycles (5 CV cycle $\sim 5$ minutes at $100 \mathrm{mV} / \mathrm{s}$ ). To study long term diffusion of Dex, if any, UV measurements were also taken after a 24-hour soaking.

\subsubsection{Ultraviolet (UV) Spectroscopy}

The released drug was detected using UV spectrometer (Genesys $5^{\mathrm{TM}}$ spectrophotometer, Thermospectronic). The readings were taken at $242 \mathrm{~nm}$, the characteristic absorbance band of Dex. Double distilled water was used as blank and the reading of PBS alone was subtracted from the reading for released sample. A standard calibration curve for Dex was plotted to define the quantitative relationship between observed absorbance and concentration of Dex.

\subsubsection{Impedance Measurements}

Electrochemical impedance spectroscopy was done using the Gamry Potentiostat, FAS2/Femostat, under the control of the Gamry Framework software. A solution of 0.1 M PBS buffer $(25 \mathrm{ml})(\mathrm{pH}=7.2)$ was used as the electrolyte in a three-electrode cell. A platinum foil was used as the counter electrode, a saturated calomel electrode (SCE) was used as the reference electrode and the gold coated electrode with or without polymer coatings was used as the working electrode. AC sinusoid signal of $5 \mathrm{mV}$ amplitude, with the $\mathrm{DC}$ potential set to $0 \mathrm{~V}$ was 
used as the input. The value of the impedance was determined at four discrete frequencies per decade over the range of 10 to $10^{5} \mathrm{~Hz}$.

\subsubsection{Statistical Analysis}

Statistical analysis was performed in SPSS. For comparisons involving multiple groups, ANOVA followed by Bonferroni’s post-hoc analysis was used.

\subsection{RESULTS AND DISCUSSION}

The fundamental principle behind our drug release is explained as follows.

$$
\left[(\mathrm{PPy})^{\mathrm{x}+}\left(\mathrm{A}^{-}\right)_{\mathrm{x}}\right] \rightleftarrows \mathrm{PPy}+\mathrm{xA}^{-}
$$

Here $\mathrm{A}^{-}$is the dopant. The movement of ions in or out of the polymer film forms the basis of drug delivery with polypyrrole. In our experiments, alternating positive and negative potential was applied using cyclic voltammetry to release the anionic Dex from the PPy-Dex film. The change of oxidation state is supported by the cyclic voltammogram (figure 5). Cyclic voltammetry provides information about the intrinsic redox reaction of the electrode materials as the potential of the electrode is swept in a cyclic manner. The cyclic voltammogram is a plot of the measured current against the applied voltage at the working electrode vs. the reference electrode. Polypyrrole is known to have switching properties and this is evident by the appearance of characteristic anodic and cathodic peaks as seen in figure 5. It is to be noted that during the first 2-3 CV cycles these peaks were not seen. However, as the number of CV cycles 
increase, the peaks become evident. The figure shows appearance of characteristic oxidation and reduction peaks at $+0.49 \mathrm{~V}$ and $-0.34 \mathrm{~V}$ respectively. The reduction peak is an indication of the release of the drug. The absence of peaks in the first 2-3 cycles indicated that the film needed to be rejuvenated before drug release starts.

The release of Dex in solution was quantified using UV spectrometry at $242 \mathrm{~nm}$. The released amount triggered by $\mathrm{CV}$ stimulus is displayed in figure 6. It is evident from the graph that the release of Dex correlated in roughly linear fashion $\left(R^{2}=0.9964\right)$ with the number of CV stimulus in the given $\mathrm{CV}$ cycle range. As a control, UV readings were taken from coated electrodes dipped in PBS to which no electrical stimulus was applied. As expected, there was no significant release of Dex from these control samples by diffusion. As diffusion is a time dependant process, we also took readings after 24 hours and saw no significant Dex release. The above observation indicates that our system is a true electrically controlled release system.

Estimates of the amount of drug deposited in the film were obtained using the following governing equation [46]

$$
\begin{aligned}
& P y \rightarrow(P P y)^{x+}+2 H^{+}+(2+x) e^{-} \\
& \frac{x}{2+x}=\frac{Q_{\text {cap }}}{Q_{\text {dep }}}
\end{aligned}
$$

where, $\mathrm{Q}_{\text {dep }}$ is the deposition charge and $\mathrm{Q}_{\text {cap }}$ is the charge capacity under $1 \mathrm{CV}$ cycle. For $\mathrm{Q}_{\text {dep }}=20 \mathrm{mC}$ and $\mathrm{Q}_{\text {cap }}=2.5 \mathrm{mC}$, we can load nearly $17.4 \mu \mathrm{g} / \mathrm{cm}^{2}$ of Dex in the PPy-Dex film. We are thus able to achieve $92 \%$ drug release at the end of $30 \mathrm{CV}$ cycles.

Previous research has shown that dexamethasone is effective at a concentration of 0.2-0.7 $\mu \mathrm{M}$ [47] and around this local concentration, pronounced reductions in inflammatory tissue reactions are seen around neural implants. We are able to release $0.5 \mu \mathrm{g} / \mathrm{cm}^{2}$ Dex after each CV 
cycle and to a total of nearly $16 \mu \mathrm{g} / \mathrm{cm}^{2}$ after $30 \mathrm{CV}$ cycles. Based on most of the histological studies, the reactive region indicated by enhanced glial fibrillary acidic protein (GFAP), a key intermediate filament, activity around the neural electrode arrays has a radius of less than 500 $\mu \mathrm{m}[7,48]$. A release of $0.5 \mu \mathrm{g} / \mathrm{cm}^{2}$ Dex will result in average Dex concentration of $1 \mu \mathrm{M}$ within $500 \mu \mathrm{m}$ radius from the electrode. Thus, 1 dose triggered by $1 \mathrm{CV}$ may be sufficient to reduce the inflammation and with successive CV cycles the concentration of Dex remains in the $\mu \mathrm{M}$ range and can be expected to eliminate adverse tissue reaction to the electrode in long term. Certainly, the in vivo situation may be different from our theoretical estimation. The actual efficiency and appropriate dosage of Dex will only be determined in the in vivo studies.

It should be pointed out that we also examined the UV absorption at $210 \mathrm{~nm}$, the maximum UV absorbance band of pyrrole, and found that there was a minute amount of pyrrole monomer in the solution after the release. Fortunately, the contribution of pyrrole to the $242 \mathrm{~nm}$ absorbance, used for quantification of Dex, was negligible. The in vitro data also demonstrated that the leached pyrrole was not toxic to the cultured cells.

Most of the previously reported polypyrrole based molecule release systems utilize step potential stimuli (positive or negative depending on the charge of the ions to be released). The released dosage was found to be a function of time (time of applied potential stimuli). The step function was used initially in our preliminary studies, and was found to be less effective than cyclic potential stimuli towards drug release. The two release mechanisms will be analyzed and compared as follows.

In the case of step potential stimulation (for example with small anionic release), a negative potential reduces the polypyrrole, neutralizes the positive charge on the backbone and eliminates the electrostatic bonding between polypyrrole and its anionic dopants. The 
dissociation of the dopants from the polypyrrole chain may be an instant process, but the release of the anion is slow since it is driven by diffusion consisting of movement of anions from the inner film to the surface and from the surface to the solution. For dense films, as in our studies, the former is much slower, and hence the quick release is mainly from the surface, and the bulk release may take hours. In addition, under the applied negative potential, the reduced film will become electrically non-conductive. The longer the film is held at a negative potential, the more difficult it is to recover its conductivity after the stimulus is removed. Such a system is not ideal in most of the practical application when electrodes have other functions to perform.

In the case of cyclic potential stimulation, the film undergoes a fast swelling and deswelling process caused by ion and water movement in and out of the film [49-51]. This process may act as a pump to push the anions out and dramatically speed up the bulk release (seconds or minutes). When the potential is being swept at a high speeds $(100 \mathrm{mV} / \mathrm{sec}$, in our case), only a portion of the dopants are dissociated and pumped out during each cycle. This forms the basis of linear correlation between released dose and number of cyclic stimulus, ideal for on command dose controlled release. In addition, without application of a long potential bias, the polymer film can remain conductive after each release stimulus, consequently the electrode remains functional.

Several observations provide support for the above mechanism. First, a closer look at the deposited film surface before and after release was taken under SEM. Figure 7 shows that before release the film appears essentially smooth. After $50 \mathrm{CV}$ cycles, however, there were appearance of cracks. This breakdown of the film starts to appear after $\sim 30 \mathrm{CV}$ cycles. This suggests that the polymer-drug film indeed undergoes physical changes of swelling and deswelling during the $\mathrm{CV}$ cycles which causes its delamination and breakdown. Also, higher 
charge density resulted in rougher films (shown in figure 4b) but not higher drug release, which further provides evidence that the drug release triggered by the electrical stimulus (CV) occurs from within the film and was not a surface phenomenon.

The film morphology before and after CV stimulated release was also analyzed using optical microscopy, as shown in figure 8. A change in color of the film induced by CV cycles was observed and it appeared to be darker in color than the original PPy-Dex film. The color of the film reflects its oxidative state and configuration of the polymer/dopants complex. This color change indicated that the film underwent structural and compositional changes during the course of CV treatment and the accompanying Dex release.

The electrical properties of the film were evaluated using impedance measurements. Impedance is a complex quantity defined as $\mathrm{Z}(\omega) \equiv|\mathrm{Z}(\omega)| \mathrm{e}^{\mathrm{i} \theta(\omega)} \equiv \mathrm{v}(\omega) / \mathrm{i}(\omega)$, where $\mathrm{v}(\omega)$ is the transform of an alternating potential signal applied to the system and i $(\omega)$ is the transform of the alternating current signal that results. The impedance is characterized by a magnitude $(|Z|)$ and phase angle $(\theta)$, and is generally represented as a function of frequency. Impedance spectroscopy involves measuring the electrode impedance over a spectrum of frequencies. Impedance spectroscopy is often used in neural electrode analysis for characterizing electrode/tissue or electrode/solution interface. So it is a relevant technique to be used here to examine the effect of deposited PPy/Dex on the bare electrode. The results are displayed in figure 9. The impedance of the freshly made coated electrode is higher than the uncoated gold electrode at all frequencies. However, the impedance dropped immediately after the first few CV cycles. The impedance magnitude was lower than that of gold in the lower frequency range and it continued to be lower for the rest of the $30 \mathrm{CV}$ cycles. It appears that the polymer-drug film is not as conductive initially, and perhaps was left at a reduced state immediately after 
deposition. Rejuvenation through a few $\mathrm{CV}$ cycles can activate the film and make it more conductive. This rejuvenation may result from exchange of bulky Dex molecules with smaller anions like $\mathrm{H}_{2} \mathrm{PO}_{4}{ }^{-}$and $\mathrm{HPO}_{4}{ }^{2-}$, and $\mathrm{Cl}^{-}$from $\mathrm{PBS}$, causing an increase in conductivity of the deposited film. Once in the more conductive state, the impedance becomes lower than bare gold, a commonly seen phenomenon in conducting polymer electrodes due to the higher surface area and more efficient charge transport of the polymer coating [52, 53]. This is important since having low impedance is beneficial in recording minute extracellular neural signals.

\subsection{CONCLUSION}

Dex release from PPy-Dex films on gold coated coverslips was carried out using CV technique. A linear release profile was seen on application of successive CV cycles and we were able to successfully release $92 \%$ of the incorporated drug into PBS after $30 \mathrm{CV}$ cycles. Surface characterization using SEM demonstrated that the polymer film starts to break down beyond 30 CV cycle. The optical microscopy images show change in color of the polymer film before and after drug release, indicating the structural and chemical changes in the film due to application of CV stimuli. Impedance studies also supported these observations, as indicated by lowered impedance after application of CV stimuli. 


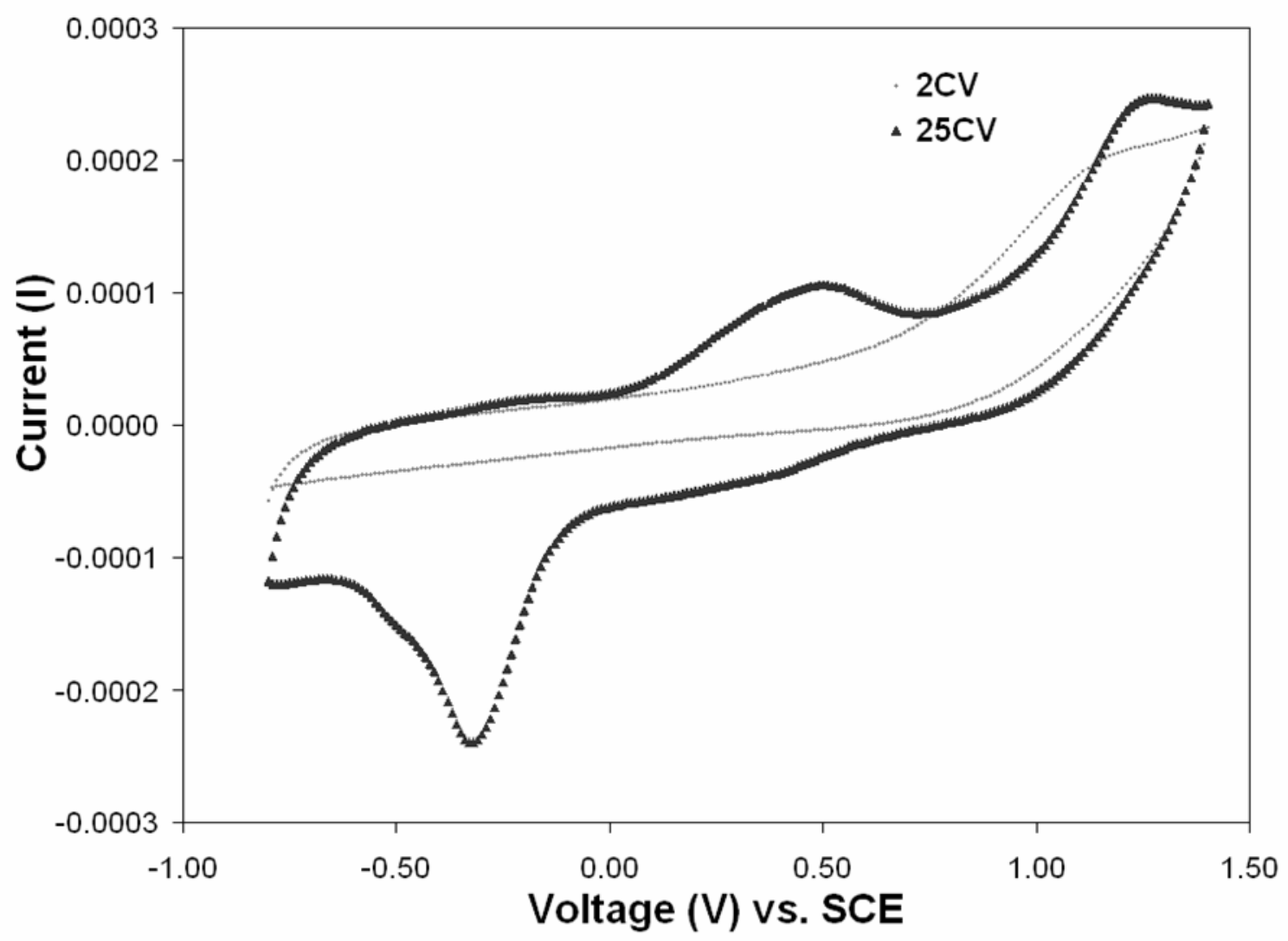

Figure 5. Characteristic Cyclic Voltammogram.

At the $2^{\text {nd }} \mathrm{CV}$ cycle no peaks are observed, implying there is no sudden release of drug and that the first few cycles are used to 'rejuvenate the film'. Anodic and cathodic peaks begin appearing in the following CV cycles. At $25 \mathrm{CV}$ cycle, characteristic oxidation peak (anions move in) (a) and reduction peak (anions including Dex move out) (b) are evident. 


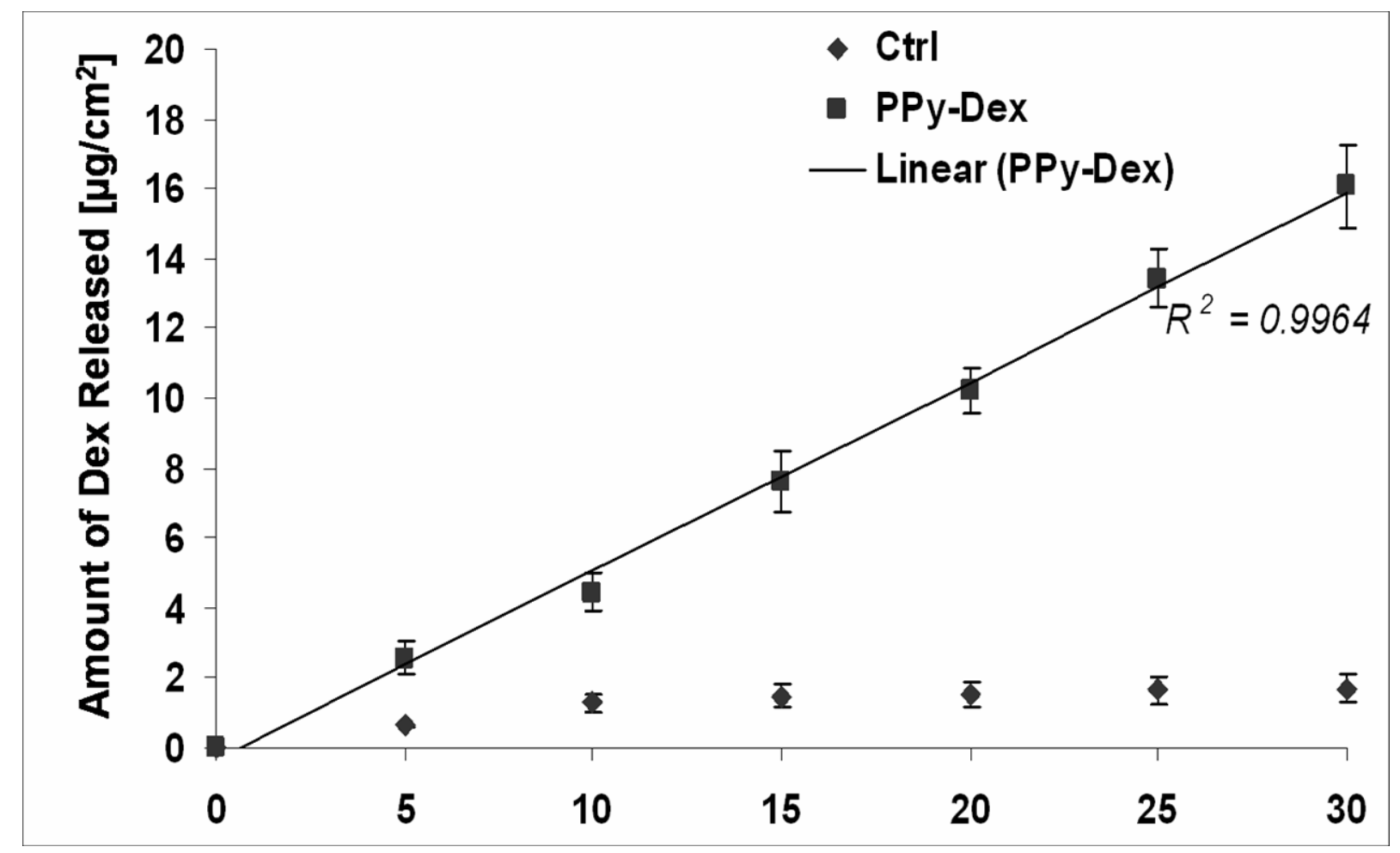

Figure 6. Release profile of Dex from the polymer-drug coated electrodes.

The release of Dex $(n=5, \pm$ SEM) was detected using UV spectroscopy at $242 \mathrm{~nm}$. The drug release is approximately linear. For PPy-Dex, the x-axis represents number of CV cycles. Control was set up to study the release from the film in the absence of any electrical stimulation. In this case the $\mathrm{x}$-axis represents the number of minutes (at $100 \mathrm{mV} / \mathrm{sec}$ scan rate, $1 \mathrm{CV}$ cycle between $-0.8 \mathrm{~V}$ to $1.4 \mathrm{~V}$ takes approximately 1 minute). There is negligible release, possibly due to diffusion of loosely attached dex from the surface. 

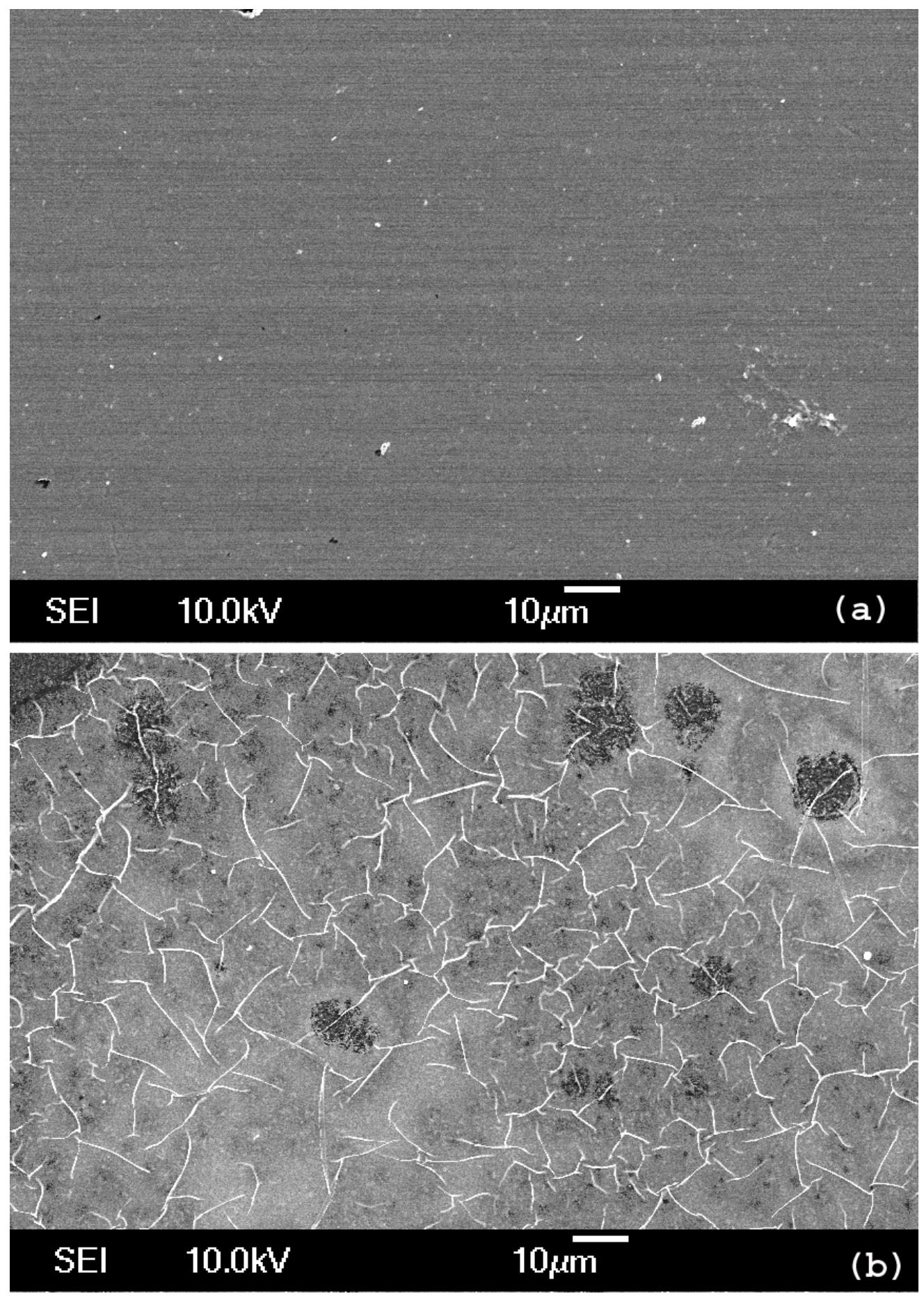

\section{Figure 7. SEM images}

(a) PPy-Dex coated film (b) PPy-Dex film after $50 \mathrm{CV}$ cycles. The SEM shows smooth surface for the deposited films. Cracks become evident after $\sim 30 \mathrm{CV}$ cycles. These are prominently seen in (b) after $50 \mathrm{CV}$ cycle. 


\section{PPy-Dex coating}

\section{PPy-Dex after CV}

Figure 8. Enface image of the gold polymer interface under optical microscope

Initial CV cycles rejuvenate the polymer surface and the surface appears darker in color than the original PPy-Dex coating. The dark color corresponds to oxidized state of the film. This change in color reflects a change in the chemical configuration of the polymer film. 


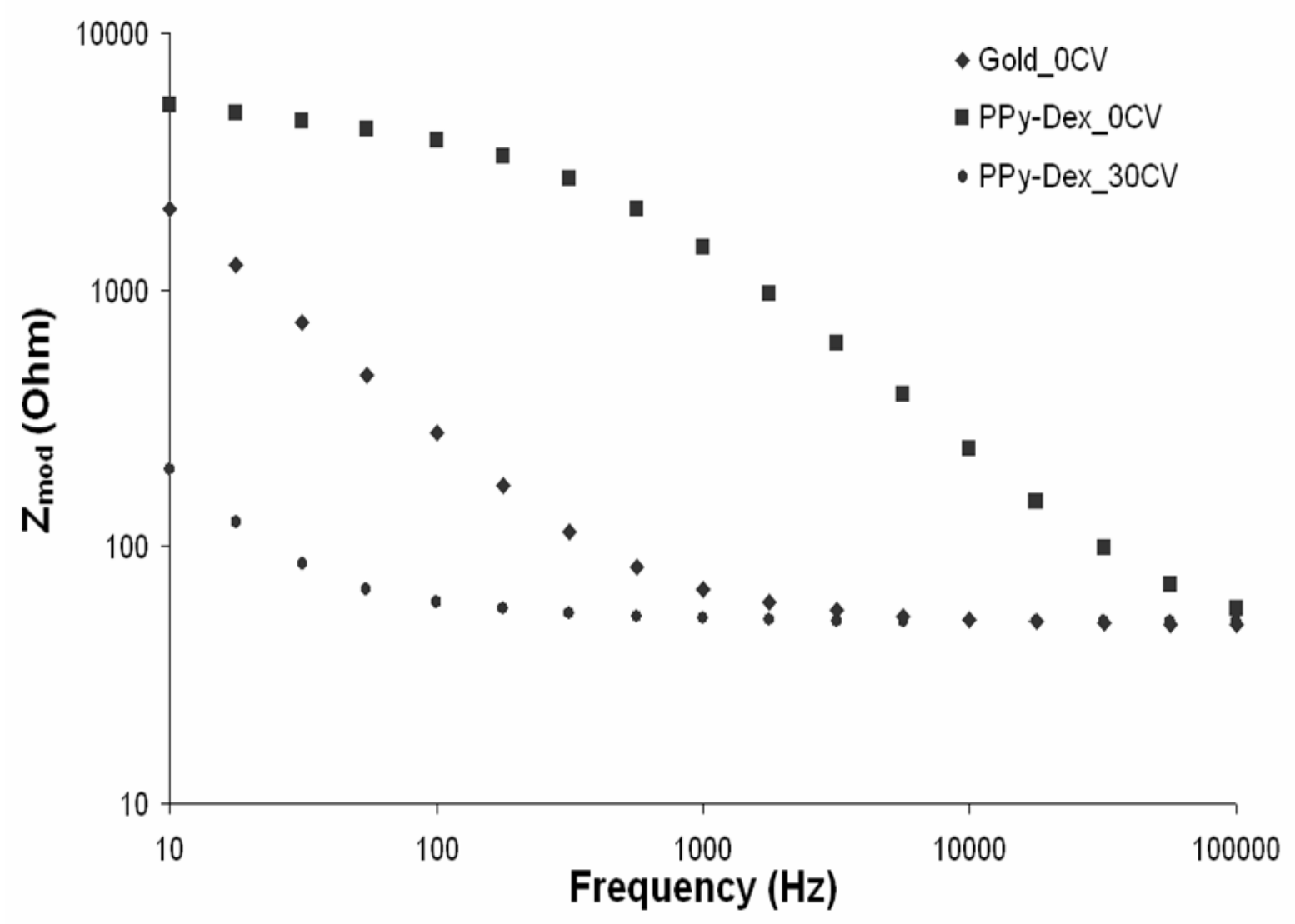

Figure 9. Impedance spectroscopy for gold and PPy-Dex film coated electrode.

Initial impedance of the film is very high. But after the first few CV cycles, the impedance drops at all frequencies and at the lower frequency range, the magnitudes are lower than those of the gold electrode. This implies deposited film was in its reduced state and needed 'rejuvenation', which was provided by initial CV cycles. The lowered impedance is maintained till $30 \mathrm{CV}$ cycles. 


\subsection{IN VITRO CELL CULTURE STUDIES}

\subsection{INTRODUCTION}

After the release profile of dexamethasone from PPy-Dex films on our gold electrodes was established, testing for the efficacy of released drug was the next step. For the in vitro studies, primary murine cerebellar cells were cultured using the techniques described in this chapter. The effect of released dexamethasone on both astrocytes and neurons was studied using immunohistochemistry and the analysis was done using optical microscopy and imaging.

\subsection{MATERIALS AND METHODS}

\subsubsection{Primary neurons and astrocytes cell culture}

Primary murine neuronal cultures were prepared as described by Schnitzer et al [39]. Briefly, following trypsinization and trituration, the cells were pelleted to remove debris and resuspended in DMEM with $10 \%$ horse serum (HyClone). These resuspended cells were plated on polylysine 
(Sigma; $>400,000 \mathrm{kD}$ ) coated glass coverslips (Fisher) at a cell density of 30,000 cells per $12 \mathrm{~mm}$ diameter coverslip. Glial cultures were prepared using the protocol of Banker $\mathrm{G}$ and Goslin $\mathrm{K}$ [40]. Briefly, astrocyte and microglial cells were harvested, trypsinized, triturated and then plated using Dulbecco's modified eagle medium (DMEM), with 10\% fatal calf serum (FCS) at $37^{\circ} \mathrm{C}$ with $5 \% \mathrm{CO}_{2}$, until confluent. These glial cultures were then trypsinized and replated on polylysine-coated coverslips. The cells were allowed to settle on the coverslips for 30 minutes and treated with three groups of media - control (DMEM + 10\% FCS), Dex (DMEM +10\% FCS $+10^{-6} \mathrm{M}$ Dex) and DexR (same as Dex, except that the Dex was released in PBS from the electrode coating and then added to the media at $10^{-6} \mathrm{M}$ concentration). Cells were kept in the incubator at $37^{\circ} \mathrm{C}$ and $5 \% \mathrm{CO}_{2}$ and analyzed on day 3 for astrocytes count. The neuronal cells were treated with control, Dex and DexR media and assayed for neuronal viability and growth on day 3 .

\subsubsection{Immunocytochemistry}

Immunochemical labeling was done using antibodies against a neuronal marker, class III $\beta$-tubulin (Covance) and an astrocyte-specific marker, Glial Fibrillary Acid Protein (GFAP) (Dako). Cells were fixed with 4\% paraformaldehyde for 10 minutes. In neuronal culture, cells were treated with 1\% NP40 (CalBioChem) in 4\% paraformaldehyde for 10 minutes at room temperature. Nonspecific binding was blocked by incubation with blocking buffer $(0.5 \%$ bovine serum albumin and $10 \%$ horse serum in TBS). Primary and secondary antibodies were added for one hour each. Following immuno-staining cells were counterstained 
using the nuclear dye Hoechst $33258(2 \mathrm{mg} / \mathrm{ml}$ in PBS) (Sigma). A similar protocol was followed for staining astrocytes.

\subsubsection{Imaging and analysis}

After immunostaining, digital images were taken using the fluorescence microscope (Axioskop 2 MAT, Carl Zeiss) using AxioCamMRC5 Camera (Carl Zeiss) attached to the scope and software

AxioVision Rel 4.2. For neuronal cell culture, the neurite measurement feature of MetaMorph ${ }^{\mathrm{TM}}$ software was used. The software utilizes the values specified for various parameters i.e. cell body (width, minimum area, intensity above background), nuclei (minimum width, maximum width and intensity above background) and outgrowth (maximum width, intensity above background and minimum growth to log as significant) to accurately map the neurite outgrowth from fluorescent images. Healthy neurons were counted as those with at least one neurite and which showed co localization of $\beta$-tubulin and Hoescht stain. The cell counts on the astrocytes images was done manually by identifying GFAP staining co-localized with a Hoechst stained nuclei. For the neuronal culture, six coverslips were used for each condition (Ctrl, Dex, and DexR) and six images were taken for each coverslip at 20X magnification. For the astrocytes culture experiments, three coverslips were used for each condition and six images were taken for each coverslip at $10 \mathrm{X}$ magnification. In both cases the experiment was repeated twice. 


\subsubsection{Statistical analysis}

Statistical analysis was performed in SPSS. For comparisons involving multiple groups (astrocytes count studies), ANOVA followed by Bonferroni's post-hoc analysis was used. For the neuronal cell culture experiment, $n=6 \times 2$ and for the astrocytes culture, $n=3 \times 2$. $p$ values $<0.05$ were considered as significant.

\subsection{RESULTS AND DISCUSSION}

To study the effect and efficacy of the released Dex, in vitro studies were conducted with primary murine cerebellar glial culture. Immunostaining with antibodies against GFAP revealed lowered number of reactive astrocytes in Dex treated samples when compared to those in control media on day 3 (figure $10 \mathrm{a}-\mathrm{c}$ ). In figure $10 \mathrm{~d}$, the day 3 astrocyte counts are represented after being normalized to the day 1 control. On day 3 , the number of astrocytes in the control group almost doubled, while both the Dex added and Dex released groups showed significantly fewer astrocytes. In addition, there was no significant difference between the Dex added group and Dex released group, which implies that the released Dex was as effective as the added Dex From these studies we cannot determine if this is due to reduced proliferation or enhanced cell death. Preliminary work with microglia cells also showed lowered growth of cells, as indicated in figure 11. It is to be noted here that due to shortage of appropriate antibody for the microglia cells, the experiment with microglia cells could be done only once. 
The released Dex may serve two purposes. Firstly, it decreases the number of reactive astrocytes. Astrocytes are a major component of the insulating glial sheath formed during the inflammatory response against the electrodes, when implanted in vivo. The objective therefore was to prevent them from getting into their reactive state and our in vitro data proves that the released Dex showed such effect.

Secondly, the drug shows a positive impact on neurons at this concentration as well. Figure 12 shows the effect of Dex on the viability of neurons on day 3 for control (Ctrl) added Dex (Dex) and released Dex (DexR). There is no significant difference in the number of neurons in the control, Dex or DexR. This implies that the released drug at this concentration is not toxic to neurons. We also observed 35\% higher neurite outgrowth in cells exposed to released Dex than those in control. These observations collectively indicate that the released drug may have a neuroprotective effect. Whether dexamethasone acts directly on its receptors in neurons or indirectly through its anti-inflammatory mechanism via astrocytes and microglia is not yet clear, though literature suggests an indirect mechanism [54]. Neurodegenerative diseases like Alzheimer's disease and Parkinson's disease are often accompanied by inflammatory reactions. In vivo studies in mouse models have demonstrated that intraperitoneal injections of Dex may exert a neuroprotective effect towards injured neurons, but only in proper doses [54]. While higher doses of Dex may be toxic to neurons, a lower dose has proven to have a neuroprotective effect in those experiments. A similar mechanism may explain the higher neurite outgrowth seen in our experiments. 


\subsection{CONCLUSION}

In vitro studies were successfully conducted to study the effect of released Dex on astro-glial cells and neurons. The drug was shown to maintain its anti-inflammatory properties, upon electrochemical release from the PPy-Dex films, as seen by lowered reactive astrocyte count. Also, at the released concentration no toxic effect of Dex was seen on the cultured neurons, as indicated by healthy neuronal count. 


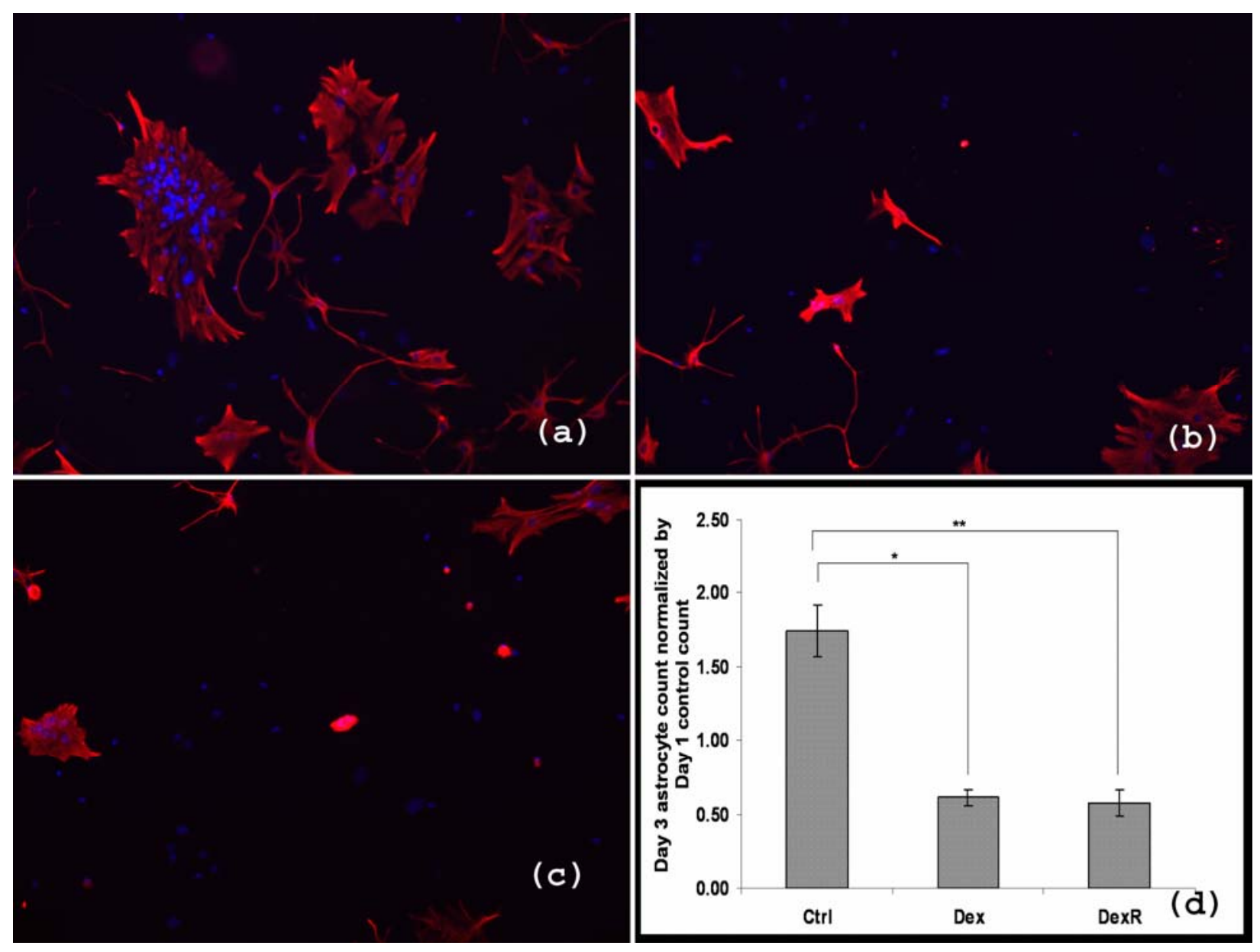

Figure 10. In-vitro studies for the effect of Dex on reactive astrocytes number

The red color is GFAP staining. The nuclei are stained using Hoechst dye. (a) Control group: no drug, (b) Dex group: Dex was added in the media at a concentration of $10^{-6} \mathrm{M}$, (c) DexR: drug was electrically released in PBS from PPy-Dex electrode and the resulting solution was added to media with the final Dex concentration of $10^{-6} \mathrm{M}$, (d) Graph showing the normalized number of astrocytes on day 3 for control (Ctrl), added Dex (Dex) and released Dex (DexR) $(\mathrm{n}=6, \pm$ SEM). Significant reduction in the number of astrocytes in both Dex and DexR media, when compared to Ctrl $\left(* \mathrm{p}<0.001\right.$ for $\mathrm{Ctrl}$ and Dex, ${ }^{* *} \mathrm{p}<0.001$ for $\mathrm{Ctrl}$ and DexR) while there was no significant difference between Dex and DexR. 

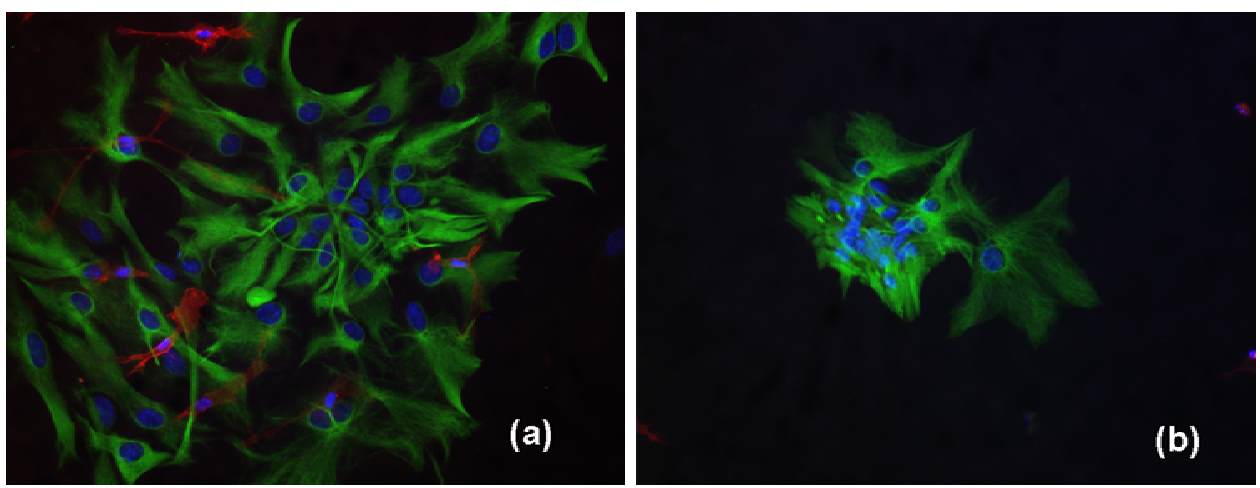

Figure 11. In-vitro studies for the effect of released Dex on astrocytes and microglia

The red color is M1/70 staining for microglia and the green is GFAP staining for astrocytes. The nuclei are stained using Hoechst dye. (a) Control group: no drug, (b) Dex group: drug was electrically released in PBS from PPy-Dex electrode and the resulting solution was added to media with the final Dex concentration of $10^{-6} \mathrm{M}$. For this one time experiment ( $\mathrm{n}=3$ samples per condition, 6 images on each sample), lowered glial cell count (astrocytes and microglia) was observed. 


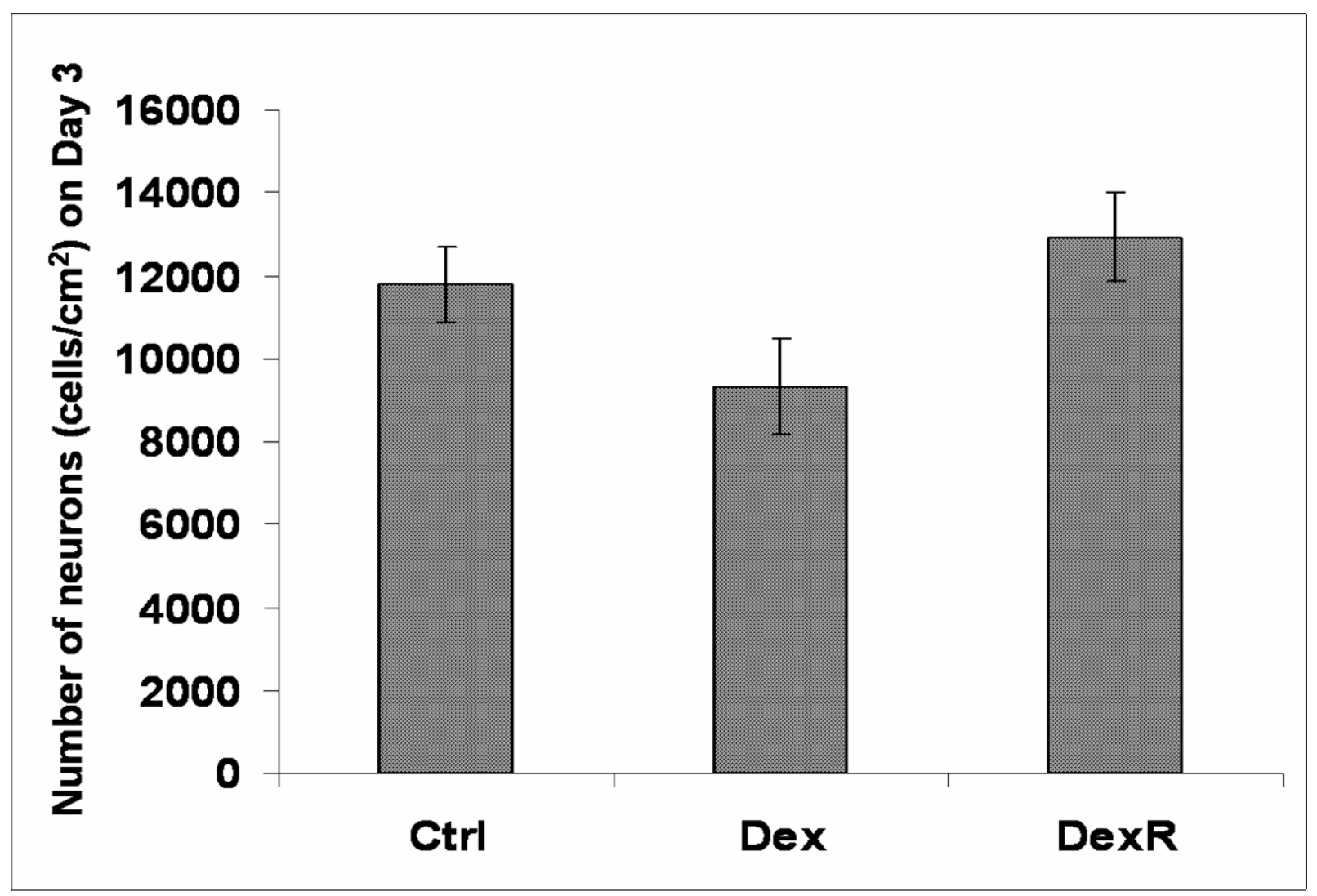

Figure 12. Effect of Dex on neuron viability on day 3 of culture.

In-vitro cultured neurons were stained using $\beta$-tubulin and Hoescht dye. Scale bar is $50 \mu \mathrm{m}$. Co-localization of $\beta$ tubulin and Hoescht was used to identify neurons. Neurite measurement, using MetaMorph TM software, enabled identification of healthy neurons as those with at least one neurite. There is no significant difference between the three groups. 


\subsection{CONCLUSION}

In this study, we have successfully developed a polypyrrole based electrode coating for electrically controlled and local delivery of an ionic form of dexamethasone (Dex), an antiinflammatory synthetic glucocorticoid. An appealing feature of the system is that it allows local drug release from individual electrode coatings in a controlled manner. Initial results show that out of a $50 \mathrm{~nm}$ thick PPy/Dex film, we are able to release $0.5 \mu \mathrm{g} / \mathrm{cm}^{2}$ Dex after each CV cycle and upto $92 \%$ release after $30 \mathrm{CV}$ cycles. In vitro studies on murine glial cells suggest that the released drug effectively reduces the number of reactive astrocytes and microglia. In addition, release of drug from the polymer coating does not produce any toxic side effects as seen by the healthy neuronal growth. In fact, the released drug caused increased neurite formation when compared to control.

In summary, we have established a drug delivery system that allows on-command, precise and local release of drugs from neural electrodes. The release dosage can be controlled via controlling the applied electrical stimuli. The mechanism is not confined to this particular polymer-drug system and may be extended to other combinations of conducting polymer and suitable dopants. Furthermore, this system can be easily integrated into any implantable electrode device setup, that are used clinically (such as deep brain stimulators and cochlear implants) or in a research setting (other neural prosthetic devices that are being actively 
developed). The electrical command for release control can be conducted via the preexisting circuits.

Further studies can be done to improve the system developed in this project. The current polymer polypyrrole allows consistent release for $\sim 30 \mathrm{CV}$ cycles, following which the film does not remain stable. While such a lifetime maybe sufficient in some applications, one may need to improve the stability of the polymer for prolonged release applications. This may be improved in the following ways. Firstly, an intrinsically more chemically and mechanically stable conducting polymer, like polyethylenedioxythiophene (PEDOT) can be selected. Secondly, the mechanical stability of the polymer and adherence between polymer and electrode substrate maybe enhanced. For example, addition of filler molecules or physical crosslinkers could stabilize the polymer film. To increase the adherence between the polymer and electrode substrate, modifications on the surface and in the original monomer can be done, which in turn help to form covalent binding between the polymer and electrode surface [55]. Alternatively the adherence can be improved by roughening the surface of the electrode [56].

The next ideal step after in-vitro cell culture studies would be to study the release of the drug in vivo from implanted neural devices. While our CV and Dex release data suggests that most of the dex was incorporated as dopants and will not be released without electrical perturbance, we can not exclude the possible material degradation in vivo in long term which could lead to release without electrical command. This may be evaluated through in vivo degradation study.

Another aspect worth investigating would be how to increase drug load and the release efficiency. Research has shown $[57,58]$ that it is possible to electrochemically synthesize conducting polymers inside a hydrogel support matrix. Such a matrix offers the obvious 
advantages of higher water content and surface area due to 3D porous nature which allow more drug incorporation and faster release. Surface area can also be greatly enhanced by creating nano-fibrous morphology through various nano-templating techniques [59-61]. 


\section{BIBLIOGRAPHY}

[1] W. F. Windle, C. D. Clemente and W. W. Chambers, Inhibition of formation of a glial barrier as a means of permitting a peripheral nerve to grow into the brain, J Comp. Neurol. 96 (2) (1952) 359-369.

[2] T. Roitbak and E. Sykova, Diffusion barriers evoked in the rat cortex by reactive astrogliosis, Glia 28 (1) (1999) 40-48.

[3] W. J. Brown, T. L. Babb, H. V. Soper, J. P. Lieb, C. A. Ottino and P. H. Crandall, Tissue reactions to long-term electrical stimulation of the cerebellum in monkeys, J. Neurosurg. 47 (3) (1977) 366-379.

[4] A. F. Reynolds and A. G. Shetter, Scarring around cervical epidural stimulating electrode., Neurosurgery 13 ((1)) (1983) 63-65.

[5] D. J. Edell, V. V. Toi, V. M. Mcneil and L. D. Clark, Factors Influencing the Biocompatibility of Insertable Silicon Microshafts in Cerebral-Cortex, IEEE. Trans. Biomed. Eng. 39 (6) (1992) 635-643.

[6] C. Schmidt, A. Horwitz, D. Lauffenburger and M. Sheetz, Integrin-cytoskeletal interactions in migrating fibroblasts are dynamic, asymmetric, and regulated, J. Cell Biol. 123 (4) (1993) 977-991.

[7] J. N. Turner, W. Shain, D. H. Szarowski, M. Andersen, S. Martins, M. Isaacson and H. Craighead, Cerebral astrocyte response to micromachined silicon implants, Exp. Neurol. 156 (1) (1999) 33-49.

[8] A. C. Hoogerwerf and K. D. Wise, A three-dimensional microelectrode array for chronic neural recording, IEEE. Trans. Biomed. Eng. 41 (12) (1994) 1136-1146.

[9] X. Cui, J. Wiler, M. Dzaman, R. A. Altschuler and D. C. Martin, In vivo studies of polypyrrole/peptide coated neural probes, Biomaterials 24 (5) (2003) 777-787.

[10] M. J. Ignatius, N. Sawhney, A. Gupta, B. M. Thibadeau, O. R. Monteiro and I. G. Brown, Bioactive surface coatings for nanoscale instruments: effects on CNS neurons, $J$ Biomed Mater Res 40 (2) (1998) 264-274. 
[11] L. Kam, W. Shain, J. N. Turner and R. Bizios, Selective adhesion of astrocytes to surfaces modified with immobilized peptides, Biomaterials 23 (2) (2002) 511-515.

[12] Y. Zhong, X. Yu, R. Gilbert and R. Bellamkonda, Stabilizing electrode-host interfaces: a tissue engineering approach, Journal of rehabilitation research and development 38 (6) (2001) 667-632.

[13] K. Webb, E. Budko, T. J. Neuberger, S. Chen, M. Schachner and P. A. Tresco, Substratebound human recombinant L1 selectively promotes neuronal attachment and outgrowth in the presence of astrocytes and fibroblasts, Biomaterials 22 (10) (2001) 1017-1028.

[14] R. Biran, K. Webb, M. D. Noble and P. A. Tresco, Surfactant-immobilized fibronectin enhances bioactivity and regulates sensory neurite outgrowth, Journal of Biomedical Materials Research 55 (1) (2001) 1-12.

[15] A. F. Mensinger, D. J. Anderson, C. J. Buchko, M. A. Johnson, D. C. Martin, P. A. Tresco, R. B. Silver and S. M. Highstein, Chronic recording of regenerating VIIIth nerve axons with a sieve electrode, Journal of Neurophysiology 83 (1) (2000) 611-615.

[16] P. R. Kennedy, S. S. Mirra and R. A. E. Bakay, The Cone Electrode - Ultrastructural Studies Following Long-Term Recording in Rat and Monkey Cortex, Neuroscience Letters 142 (1) (1992) 89-94.

[17] P. R. Kennedy, R. A. E. Bakay and S. M. Sharpe, Behavioral-Correlates of ActionPotentials Recorded Chronically inside the Cone Electrode, Neuroreport 3 (7) (1992) 605-608.

[18] M. Nishi, N. Takenaka, N. Morita, T. Ito, H. Ozawa and M. Kawata, Real-time imaging of glucocorticoid receptor dynamics in living neurons and glial cells in comparison with non-neural cells, Eur J Neurosci 11 (6) (1999) 1927-1936.

[19] K. L. Crossin, M. H. Tai, L. A. Krushel, V. P. Mauro and G. M. Edelman, Glucocorticoid receptor pathways are involved in the inhibition of astrocyte proliferation, Proc Natl Acad Sci U S A 94 (6) (1997) 2687-2692.

[20] L. Minghetti, A. Nicolini, E. Polazzi, A. Greco, M. Perretti, L. Parente and G. Levi, Downregulation of microglial cyclo-oxygenase-2 and inducible nitric oxide synthase expression by lipocortin 1, Br J Pharmacol 126 (6) (1999) 1307-1314.

[21] R. Kiefer and G. W. Kreutzberg, Effects of dexamethasone on microglial activation in vivo: selective downregulation of major histocompatibility complex class II expression in regenerating facial nucleus, J Neuroimmunol 34 (2-3) (1991) 99-108.

[22] J. N. Turner, W. Shain, D. H. Szarowski, M. Andersen, S. Martins, M. Isaacson and H. Craighead, Cerebral astrocyte response to micromachined silicon implants, Exp Neurol 156 (1) (1999) 33-49. 
[23] D. Kim, Martin, D.C.,Sustained release using PLGA nanoparticles coated on neural prosthetic devices, Society for biomaterials 30th annual meeting transactions (2005).

[24] Y. Zhong, McConnell G., Bellamkonda R.V.,In vitro and In vivo study of a novel drug releaseing coating for implanted neural electrodes, Neural Interfaces Workshop November (2004).

[25] S. T. Retterer, K. L. Smith, C. S. Bjornsson, K. B. Neeves, A. J. H. Spence, J. N. Turner, W. Shain and M. S. Isaacson, Model neural prostheses with integrated microfluidics: A potential intervention strategy for controlling reactive cell and tissue responses, Ieee Transactions on Biomedical Engineering 51 (11) (2004) 2063-2073.

[26] G. G. Wallace, Conductive Electroactive Polymers: Intelligent Material Systems, Technomic Pub. Co., Lancaster, PA, 1997.

[27] L. L. Miller and X. U. Zhou, Poly(N-methylpyrrolylium) Poly(styrenesu1fonate). A Conductive, Electrically Switchable Cation Exchanger That Cathodically Binds and Anodically Releases Dopamine, Macromolecules 20 (7) (1987) 1594-1597.

[28] K. Kontturi, P. Pentti and G. Sundholm, Polypyrrole as a model membrane for drug delivery, Journal of Electroanalytical Chemistry 453 (1-2) (1998) 231-238.

[29] B. Massoumi and A. A. Entezami, Electrochemically stimulated 2-ethylhexyl phosphate (EHP) release through redox switching of conducting polypyrrole film and polypyrrole/poly ( -methylpyrrole) or self-doped polyaniline bilayers, Polymer International 51 (2002) 555-560.

[30] J. M. Pernaut and J. R. Reynolds, Use of conducting electroactive polymers for drug delivery and sensing of bioactive molecules. A redox chemistry approach, Journal of Physical Chemistry B 104 (17) (2000) 4080-4090.

[31] C. E. Schmidt, V. R. Shastri, J. P. Vacanti and R. Langer, Stimulation of neurite outgrowth using an electrically conducting polymer, Proceedings of the National Academy of Sciences of the United States of America 94 (17) (1997) 8948-8953.

[32] J. Y. Wong, R. Langer and D. E. Ingber, Electrically conducting polymers can noninvasively control the shape and growth of mammalian cells., Proc Natl Acad Sci U S A. . 91 (8) (1994) 3201-3204.

[33] P. M. George, A. W. Lyckman, D. A. LaVan, A. Hegde, Y. Leung, R. Avasare, C. Testa, P. M. Alexander, R. Langer and M. Sur, Fabrication and biocompatibility of polypyrrole implants suitable for neural prosthetics, Biomaterials 26 (17) (2005) 3511-3519.

[34] T. Aoki, M. Tanino, K. Sanui, N. Ogata, K. Kumakura, T. Okano, Y. Sakurai and M. Watanabe, Culture of Mammalian-Cells on Polypyrrole-Coated Ito as a Biocompatible Electrode, Synthetic Metals 71 (1-3) (1995) 2229-2230. 
[35] J. Y. Wong, R. Langer and D. E. Ingber, Electrically Conducting Polymers Can Noninvasively Control the Shape and Growth of Mammalian-Cells, Proceedings of the National Academy of Sciences of the United States of America 91 (8) (1994) 3201-3204.

[36] A. B. Smith, Knowles, C.J., Potential Role of a Conducting Polymer in Biochemistry Protein Binding Properties, Biotechnol. Appl. Biochem 12 (6) (1990) 661-669.

[37] X. Y. Cui, V. A. Lee, Y. Raphael, J. A. Wiler, J. F. Hetke, D. J. Anderson and D. C. Martin, Surface modification of neural recording electrodes with conducting polymer/biomolecule blends, Journal of Biomedical Materials Research 56 (2) (2001) 261-272.

[38] T. M. Herne and M. J. Tarlov, Characterization of DNA probes immobilized on gold surfaces, Journal of the American Chemical Society 119 (38) (1997) 8916-8920.

[39] J. Schnitzer and M. Schachner, Characterization of isolated mouse cerebellar cell populations in vitro, J Neuroimmunol 1 (4) (1981) 457-470.

[40] G. Banker and K. Goslin, Culturing Nerve Cells, MIT Press, Cambridge, MA, 1998.

[41] J. S. Kroin, R. B. Schaefer and R. D. Penn, Chronic intrathecal administration of dexamethasone sodium phosphate: Pharmacokinetics and neurotoxicity in an animal model, Neurosurgery 46 (1) (2000) 178-182.

[42] N. S. Allen, K. S. Murray, R. J. Fleming and B. R. Saunders, Physical properties of polypyrrole films containing trisoxalatometallate anions and prepared from aqueous solution, Synthetic Metals 87 (3) (1997) 237-247.

[43] X. Cui and D. C. Martin, Fuzzy gold electrodes for lowering impedance and improving adhesion with electrodeposited conducting polymer films, Sensors and Actuators A: Phys. 103 (3) (2003) 384-394.

[44] R. Kostic, D. Rakovic, S. A. Stepanyan, I. E. Davidova and L. A. Gribov, Vibrational Spectroscopy of Polypyrrole, Theoretical-Study, Journal of Chemical Physics 102 (8) (1995) 3104-3109.

[45] K. Warriner, S. Higson, D. Ashworth, I. Christie and P. Vadgama, Stability of dodecyl sulphate-doped poly(pyrrole) glucose oxidase modified electrodes exposed in human blood serum, Materials Science \& Engineering C-Biomimetic Materials Sensors and Systems 5 (2) (1997) 81-90.

[46] R. John and G. G. Wallace, The use of microelectrodes to probe the electropolymerization mechanism of heterocyclic conducting polymers, Journal of Electroanalytical Chemistry 306 (1-2) (1991) 157-167.

[47] W. Shain, L. Spataro, J. Dilgen, K. Haverstick, S. Retterer, M. Isaacson, M. Saltzman and J. $\mathrm{N}$. Turner, Controlling cellular reactive responses around neural prosthetic devices using 
peripheral and local intervention strategies, Ieee Transactions on Neural Systems and Rehabilitation Engineering 11 (2) (2003) 186-188.

[48] D. H. Szarowski, M. D. Andersen, S. Retterer, A. J. Spence, M. Isaacson, H. G. Craighead, J. N. Turner and W. Shain, Brain responses to micro-machined silicon devices, Brain Res. 983 (1-2) (2003) 23-35.

[49] A. S. Hutchison, T. W. Lewis, S. E. Moulton, G. M. Spinks and G. G. Wallace, Development of polypyrrole-based electromechanical actuators, Synthetic Metals 113 (12) (2000) 121-127.

[50] E. W. H. Jager, E. Smela, O. Inganas and I. Lundstrom, Polypyrrole microactuators, Synthetic Metals 102 (1-3) (1999) 1309-1310.

[51] J. D. Madden, R. A. Cush, T. S. Kanigan and I. W. Hunter, Fast contracting polypyrrole actuators, Synthetic Metals 113 (1-2) (2000) 185-192.

[52] X. Y. Cui, J. F. Hetke, J. A. Wiler, D. J. Anderson and D. C. Martin, Electrochemical deposition and characterization of conducting polymer polypyrrole/PSS on multichannel neural probes, Sensors and Actuators a-Physical 93 (1) (2001) 8-18.

[53] Y. H. Xiao, X. Y. Cui, J. M. Hancock, M. B. Bouguettaya, J. R. Reynolds and D. C. Martin, Electrochemical polymerization of poly(hydroxymethylated-3,4-ethylenedioxythiophene) (PEDOT-MeOH) on multichannel neural probes, Sensors and Actuators B-Chemical 99 (2-3) (2004) 437-443.

[54] I. Kurkowska-Jastrzebska, T. Litwin, I. Joniec, A. Ciesielska, A. Przybylkowski, A. Czlonkowski and A. Czlonkowska, Dexamethasone protects against dopaminergic neurons damage in a mouse model of Parkinson's disease, Int Immunopharmacol 4 (1011) (2004) 1307-1318.

[55] E. Smela, Thiol-modified pyrrole monomers: 4. Electrochemical deposition of polypyrrole over 1-(2-thioethyl)pyrrole, Langmuir 14 (11) (1998) 2996-3002.

[56] X. Cui and D. C. Martin, Fuzzy gold electrodes for lowering impedance and improving adhesion with electrodeposited conducting polymer films, Sensors and Actuators A: Physical 103 (3) (2003) 384-394.

[57] B. C. Kim, G. M. Spinks, G. G. Wallace and R. John, Electroformation of conducting polymers in a hydrogel support matrix, Polymer 41 (5) (2000) 1783-1790.

[58] D. H. Kim, M. Abidian and D. C. Martin, Conducting polymers grown in hydrogel scaffolds coated on neural prosthetic devices, Journal of Biomedical Materials Research Part A 71A (4) (2004) 577-585.

[59] S. Ghosh and O. Inganas, Nano-structured conducting polymer network based on PEDOTPSS, Synthetic Metals 121 (1-3) (2001) 1321-1322. 
[60] J. F. Hulvat and S. I. Stupp, Liquid-crystal templating of conducting polymers, Angewandte Chemie-International Edition 42 (7) (2003) 778-781.

[61] J. F. Hulvat and S. I. Stupp, Anisotropic properties of conducting polymers prepared by liquid crystal templating, Advanced Materials 16 (7) (2004) 589-+. 